\title{
A importância da assistência de enfermagem no cateterismo cardíaco: uma revisão
}

\section{de literatura}

\author{
The importance of nursing care in cardiac catheterization: a literature review \\ La importancia de la atención de enfermería en el cateterismo cardíaco: una revisión de la
}

literatura

Recebido: 21/11/2021 | Revisado: 29/11/2021 | Aceito: 02/12/2021 | Publicado: 04/12/2021

\author{
Raquel Ferreira de Sousa Mesquita \\ ORCID: https://orcid.org/0000-0002-4403-6270 \\ Universidade Ceuma, Brasil \\ E-mail: raquelf_sousa@ hotmail.com \\ Iracely Santos Adriao \\ ORCID: https://orcid.org/0000-0002-1103-3823 \\ Universidade Ceuma, Brasil \\ E-mail: lracelyadriao@gmail.com \\ Cristina Limeira Leite \\ ORCID: https://orcid.org/0000-0002-7321-1496 \\ Universidade Ceuma, Brasil \\ E-mail: crislimeira@gmail.com
}

\begin{abstract}
Resumo
Introdução: O cateterismo é o exame considerado padrão para diagnóstico da doença arterial coronariana, auxiliado por história clínica e sinais e sintomas, a técnica consiste em localizar o local da obstrução, avaliando o funcionamento de valvas e do coração, assim, planejando posteriormente, a melhor intervenção. Objetivo: analisar a importância da assistência de enfermagem frente ao cateterismo cardíaco. Metodologia: A pesquisa em questão tratase de uma revisão bibliográfica, descritiva e qualitativa, sendo fundamentada por artigos publicados em periódicos. A coleta de dados ocorreu no mês de setembro de 2021, incluído os artigos que estão dispostos nos critérios de inclusão dos últimos 5 anos, de 2016 a 2021, na língua portuguesa, disponibilizados gratuitamente nos bancos BVS, ScieELO e LILACS. Resultados: O presente estudo baseou-se em 10 artigos. Esse estudo trouxe resultados importantes tal como a importância da equipe de enfermagem, possuindo grande valor no processo de acompanhamento e monitorização, tendo em vista que ela é responsável pelo preparo prévio do material, bem como pela manutenção da monitorização e atua veementemente no processo de prevenção de agravos por meio da observação de sinais de risco. Considerações finais: Conclui-se que é de fundamental importância para reabilitação do paciente aos cuidados dos enfermeiros. Assim, pode-se dizer que uma intervenção precoce do enfermeiro é capaz de minimizar e reduzir os agravos melhorando o conforto e a segurança do paciente.
\end{abstract}

Palavras-chave: Cateterismo Cardíaco; Enfermagem; Assistência ao Paciente.

\begin{abstract}
Introduction: Cateterism is the standard examination for diagnosis of coronary artery disease, aided by clinical history and signs and symptoms, the technique is to locate the site of obstruction, evaluating the functioning of valves and heart, thus planning later on the best intervention. Purpose: to analyze the importance of nursing care in front of cardiac cateterism. Methodology: The research in question is a bibliographic, descriptive and qualitative review, based on articles published in periodicals. Data collection took place in September 2021, including articles that are set out in the criteria for inclusion of the last five years, from 2016 to 2021, in the Portuguese language, made available free of charge in BVS, ScieELO and LILACS banks. Results: This study was based on 10 articles. This study brought important results such as the importance of the nursing team, having great value in the monitoring and monitoring process, because it is responsible for preparing the material beforehand, as well as maintaining monitoring and vigorously acting in the process of preventing injury by observing risk signs. Final considerations: It is concluded that it is of fundamental importance for the rehabilitation of the patient in the care of nurses. Thus, it can be said that early intervention by the nurse is capable of minimizing and reducing staples by improving the comfort and safety of the patient.
\end{abstract}

Keywords: Cardiac Catheterization; Nursing; Patient Care.

\section{Resumen}

Introducción: El cateterismo es la prueba considerada estándar para el diagnóstico de la enfermedad arterial coronaria, ayudada por la historia clínica y los signos y síntomas, la técnica consiste en localizar el sitio de obstrucción, evaluar 
el funcionamiento de las válvulas y el corazón, planificando así posteriormente, la mejor intervención. Objetivo: analizar la importancia de los cuidados de enfermería frente al cateterismo cardíaco. Metodología: La investigación en cuestión es una revisión bibliográfica, descriptiva y cualitativa, estando basada en artículos publicados en revistas. La recolección de datos ocurrió en septiembre de 2021, incluidos los artículos que se establecen en los criterios de inclusión de los últimos 5 años, de 2016 a 2021, en portugués, disponibles de forma gratuita en los bancos BVS, ScieELO y LILACS. Resultados: El presente estudio se basó en 10 artículos. Este estudio trajo resultados importantes como la importancia del equipo de enfermería, teniendo gran valor en el proceso de monitoreo y monitoreo, considerando que es responsable de la preparación previa del material, así como de mantener el monitoreo y actúa fuertemente en el proceso de prevención de lesiones a través de la observación de señales de riesgo. Consideraciones finales: Se concluye que es de fundamental importancia para la rehabilitación del paciente al cuidado de enfermeras. Así, se puede decir que una intervención temprana de la enfermera es capaz de minimizar y reducir las lesiones, mejorando la comodidad y seguridad del paciente.

Palablas clave: Cateterismo Cardíaco; Enfermería; Atención al Paciente.

\section{Introdução}

As doenças cardiovasculares compreendem o tipo de Doenças Crônica Não Transmissíveis (DCNTs), sendo um problema de saúde de pública pelos altos gastos assistências à saúde, fazendo parte de $70 \%$ do orçamento. Mesmo diante dos avanços tecnológicos, a Doença Isquêmica Cardíaca é uma doença complexa e de risco, tendo como característica o estreitamento das artérias coronárias (Moreira et al., 2017).

O miocárdio para entrar em isquemia, passa por um longo período privado de sangue, ocorrendo a necrose do tecido por hipóxia, esse fato é chamado de Infarto Agudo do Miocárdio, sendo caracterizado pela instabilidade ocasionado pela placa aterosclerótica. A terapêutica que pode ser feita é desobstrução dessa artéria, o chamado cateterismo cardíaco, seu intuito é que coração possa retornar suas funções, reduzido complicações cardíacas. Os fatores intrínsecos ao desenvolvimento de doenças coronarianas são diversos, entre eles: tabagismo, alcoolismo, alimentação inadequada e obesidade (Maciel et al., 2016; Bertolini et al., 2019).

O cateterismo é o exame considerado padrão para diagnóstico da doença arterial coronariana, auxiliado por história clínica e sinais e sintomas, a técnica consiste em localizar o local da obstrução, avaliando o funcionamento de valvas e do coração, assim, planejando posteriormente, a melhor intervenção (Régis et al., 2017).

É um procedimento utilizado na hemodinâmica, realizado por médicos cardiologistas, enfermeiros e técnicos, sendo feito com paciente em jejum, acordado e sedado. Por ser um procedimento invasivo, os pacientes necessitam de um cuidado pré, trans e pós cateterismo (Régis et al., 2017).

Diante disso, a enfermagem tem como papel estar atento aos indicadores de piora ou desconforto dos pacientes, estando atento para solucioná-los, além de promover uma reabilitação mais rápida e humanizada. Com isso, destaca-se a importância da Sistematização da Assistência de Enfermagem (SAE) que estão pautadas para no cuidado singular, sendo direcionado e organizado ações resolutivas para problema elencado (Machado et al., 2019).

As etapas do SAE são: coleta de dados, diagnóstico de enfermagem, planejamento, implementação e avaliar toda assistência de enfermagem, denotando a importância do enfermeiro durante o cateterismo cardíaco, a fim de se atenuar e minimizar riscos, trazendo conforto ao paciente (Kuhn et al., 2015).

$\mathrm{O}$ interesse pela presente pesquisa se basear nos pouquíssimos assuntos que circundem o assunto, visto que enfermagem na hemodinâmica ainda é papel ainda muito novo e pouco explorado, sendo assim, a pergunta norteadora é: qual a importância da assistência de enfermagem frente ao cateterismo cardíaco?

O estudo pretende subsidiar novas discussões do tema, construindo novos visões, além de conceituar a função e de como a enfermagem pode auxiliar o paciente em seus anseios e dúvidas desde admissão a alta hospitalar, dinamizado o cuidado e a técnica, identificado dificuldades e percalços nesse trabalho. 
Com objetivo geral de analisar a importância da assistência de enfermagem frente ao cateterismo cardíaco, descrever o processo de cateterismo cardíaco e trabalho de enfermagem nesse procedimento, além de conceituar os cuidados com SAE ao longo e pós do cateterismo.

\section{Metodologia}

A pesquisa em questão trata-se de uma revisão bibliográfica, descritiva e qualitativa, sendo fundamentada por artigos publicados em periódicos. Abordagem qualitativa é aquela que visa tratar de um assunto não quantificável, alquilo que é subjetivo, moldado pelo meio que se encontra e descritiva por estar descrevendo um fenômeno a partir de ideias estabelecidas (Pereira et al., 2018; Marconi \& Lakatos, 2017; Severino et al., 2017; Padúa, 2019).

Para identificação do trabalho, elaborou-se a pergunta norteadora supracitada, após isso, buscou-se no DECS (Descritores em Saúde) as palavras chaves que pudessem ser utilizadas para restrição do tema, a qual elencou-se enfermagem, Cateterismo Cardíaco e Assistência ao Paciente, posteriormente com esses descritores, buscou-se na Biblioteca Virtual em Saúde (BVS) combinado os termos entre si pelo operador boleano de AND, assim, encontrou-se artigos na plataforma LILACS (Literatura Latino-Americana e do Caribe em Ciências da Saúde) e SciELO (Scientific Electronic Library Online.

A coleta de dados ocorreu no mês de setembro de 2021, incluído os artigos que estão dispostos nos critérios de inclusão dos últimos 5 anos, de 2016 a 2021, na língua portuguesa, disponibilizados gratuitamente nos bancos supracitados, excluindo todos que não estivessem acordo com pergunta da pesquisa, além de estarem em língua estrangeira e serem dos anos inferiores de 2015, e constituírem Trabalhos de Conclusão de Curso, monografia, relatos de experiência ou caso, conforme o fluxograma da pesquisa na Figura 1:

Figura 1. Fluxograma PRISMA

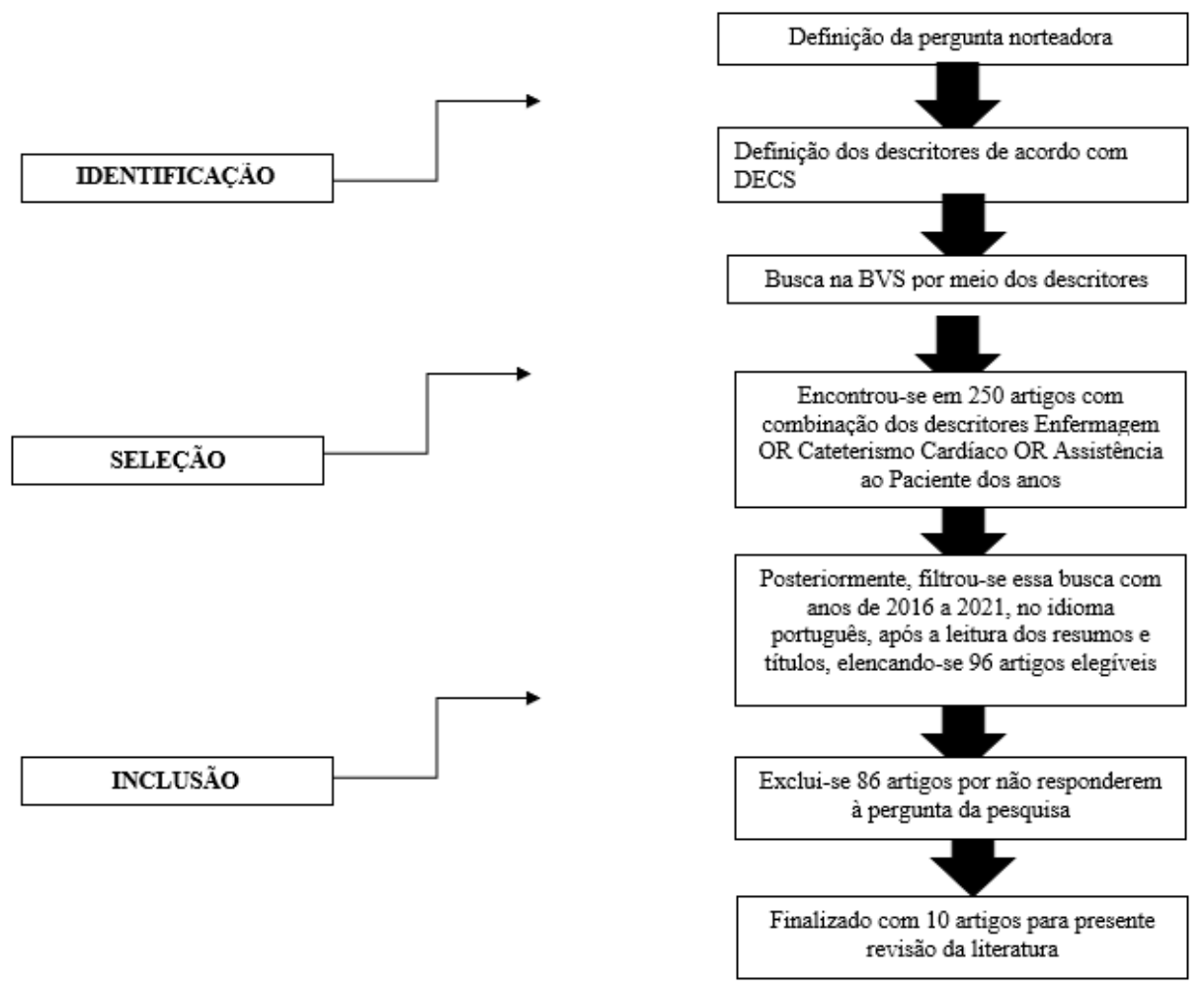




\section{Resultados}

O Quadro 1 demostrar a distribuição dos artigos pesquisados nas bases de dados BVS, LILACS e SciELO:

Quadro 1. Artigos científicos encontrados nas bases de dados selecionadas.

\begin{tabular}{|c|c|c|}
\hline BASES DE DADOS & \multicolumn{2}{c|}{ ARTIGOS } \\
\hline & ENCONTRADOS & 2 \\
\hline BVS & 70 & 6 \\
\hline SCIELO & 85 & 2 \\
\hline LILACS & 95 & INCLUS \\
\hline
\end{tabular}

Fonte: Autores (2021).

Os resultados dos artigos escolhidos para presente pesquisa estão elencados no Quadro 2, com as seguintes divisões: primeiro autor, ano, objetivo e tipo de estudo:

Quadro 2. Artigos incluídos na revisão bibliográfica.

\begin{tabular}{|c|c|c|c|c|}
\hline PRIMEIRO AUTOR & TÍTULO & ANO & OBJETIVO & TIPO DE ESTUDO \\
\hline Bianca Fontana Aguiar & $\begin{array}{l}\text { Importância dos cuidados } \\
\text { de enfermagem no } \\
\text { cateterismo cardíaco }\end{array}$ & 2016 & $\begin{array}{l}\text { Verificar as complicações ocorridas e } \\
\text { os principais cuidados de enfermagem } \\
\text { realizados antes, durante e após o } \\
\text { cateterismo cardíaco, além de traçar o } \\
\text { perfil dos pacientes submetidos ao } \\
\text { procedimento. }\end{array}$ & $\begin{array}{l}\text { Trata-se de uma } \\
\text { pesquisa retrospectiva, } \\
\text { descritiva e com } \\
\text { abordagem } \\
\text { quantitativa. }\end{array}$ \\
\hline $\begin{array}{c}\text { Tatiane Roberta Fernandes } \\
\text { Teixeira }\end{array}$ & $\begin{array}{c}\text { Compreensão de } \\
\text { pacientes às orientações } \\
\text { de enfermagem no } \\
\text { cateterismo cardíaco: uma } \\
\text { pesquisa qualitativa }\end{array}$ & 2019 & $\begin{array}{l}\text { Compreender o conhecimento dos } \\
\text { pacientes sobre o procedimento do } \\
\text { cateterismo cardíaco a fim de elaborar } \\
\text { orientações de enfermagem no pré- } \\
\text { exame }\end{array}$ & Pesquisa qualitativa. \\
\hline Andréia Avelino Oliveira & $\begin{array}{l}\text { O cateterismo cardíaco e } \\
\text { a enfermagem: a } \\
\text { importância dos } \\
\text { diagnósticos de } \\
\text { enfermagem para uma } \\
\text { assistência de qualidade }\end{array}$ & 2018 & $\begin{array}{l}\text { Evidenciar os diagnósticos de } \\
\text { enfermagem dos pacientes } \\
\text { submetidos ao cateterismo cardíaco. }\end{array}$ & Revisão integrativa. \\
\hline Juliane Lima Alencar & $\begin{array}{l}\text { Uma revisão integrativa } \\
\text { dos diagnósticos de } \\
\text { enfermagem mais } \\
\text { evidenciados no } \\
\text { cateterismo cardíaco }\end{array}$ & 2021 & $\begin{array}{c}\text { Descrever os diagnósticos de } \\
\text { enfermagem em pacientes submetidos } \\
\text { ao cateterismo cardíaco mais citados } \\
\text { na literatura. }\end{array}$ & Revisão integrativa \\
\hline $\begin{array}{c}\text { Rakel Karollyne Moreira } \\
\text { do Nascimento }\end{array}$ & $\begin{array}{l}\text { Consulta de enfermagem } \\
\text { pré-procedimento de } \\
\text { cateterismo cardíaco: } \\
\text { avaliação da satisfação do } \\
\text { paciente }\end{array}$ & 2021 & $\begin{array}{l}\text { Avaliar a satisfação dos pacientes em } \\
\text { relação à consulta de enfermagem } \\
\text { prévia ao procedimento de } \\
\text { cateterismo cardíaco. }\end{array}$ & Pesquisa quantitativo. \\
\hline $\begin{array}{l}\text { Yana Thalita Barros de } \\
\text { Oliveira Castro }\end{array}$ & $\begin{array}{c}\text { Conhecimento e } \\
\text { significado do } \\
\text { cateterismo cardíaco para } \\
\text { pacientes cardiopatas }\end{array}$ & 2016 & $\begin{array}{c}\text { Descrever o conhecimento e } \\
\text { significado do cateterismo cardíaco } \\
\text { para pacientes cardiopatas. }\end{array}$ & $\begin{array}{l}\text { Pesquisa qualitativa, } \\
\text { descritiva. }\end{array}$ \\
\hline $\begin{array}{l}\text { Vladimir Feliciano da } \\
\text { Silva }\end{array}$ & $\begin{array}{l}\text { Cateterismo cardíaco: } \\
\text { A entrevista frente aos } \\
\text { anseios do paciente }\end{array}$ & 2018 & $\begin{array}{l}\text { Avaliar de que forma está sendo } \\
\text { realizada a entrevista em pacientes } \\
\text { submetidos ao cateterismo cardíaco, } \\
\text { em um hospital de médio porte do } \\
\text { interior do Rio Grande do Sul. }\end{array}$ & $\begin{array}{l}\text { Pesquisa qualitativa, } \\
\text { transversal. }\end{array}$ \\
\hline
\end{tabular}




\begin{tabular}{|c|c|c|c|c|}
\hline $\begin{array}{c}\text { Margarida Maria da Silva } \\
\text { Soares }\end{array}$ & $\begin{array}{c}\text { Cateterismo cardíaco via } \\
\text { femoral: descrição clínica } \\
\text { e complicações } \\
\text { associadas }\end{array}$ & 2017 & $\begin{array}{c}\text { Descrever as características clínicas e } \\
\text { as complicações associadas } \\
\text { em pacientes submetidos } \\
\text { ao cateterismo cardíaco via femoral. }\end{array}$ & Pesquisa qualitativa. \\
\hline $\begin{array}{c}\text { Magda Aparecida dos } \\
\text { Santos Silva }\end{array}$ & $\begin{array}{c}\text { Relação entre orientação, } \\
\text { ansiedade e dor em } \\
\text { pacientes submetidos ao } \\
\text { cateterismo cardíaco }\end{array}$ & 2016 & $\begin{array}{c}\text { Verificar a ocorrência de sintomas de } \\
\text { ansiedade no período pré } \\
\text { intervencional de cateterismo cardí- } \\
\text { aco (CAT) e de dor nos pós CAT. }\end{array}$ & $\begin{array}{c}\text { Pesquisa qualitativa, } \\
\text { transversal. } \\
\text { Angela Antonia Sartori } \\
\text { enfermagem no setor de } \\
\text { hemodinâmica: uma } \\
\text { perspectiva adaptativa }\end{array}$ \\
enfermagem da Taxonomia II da & $\begin{array}{c}\text { Identificar os diagnósticos de } \\
\text { NANDA-I em pacientes atendidos no } \\
\text { Setor de Hemodinâmica. }\end{array}$ & $\begin{array}{c}\text { Pesquisa quantitativa, } \\
\text { transversal. }\end{array}$ \\
\hline
\end{tabular}

Fonte: Autores (2021).

\section{Discussão}

O cateterismo cardíaco, também chamado de CAT, é uma técnica invasiva, que pode ser realizada eletiva ou de urgência/emergência. Ele consiste na sedação do paciente por meio de anestesia local, posteriormente, escolhe uma punção arterial, podendo ser radial, braquial ou femoral pelo médico especializado, introduzido guia e cateter na artéria com direção a aorta e ventrículo esquerda/direito, o contraste e flurocospia tendem dá projeção as imagens das coronárias, observando as suas condições para avaliação e diagnóstico da doença coronária (Aguiar et al., 2016).

O procedimento pode ter duas abordagens.um com cateter com balão, em sua extremidade distal, que quando insuflado, comprime toda placa ateromatosa, ou com cateter com prótese de metal que é conhecida como stent acoplado ao balão para compressão total da placa, visando reestabelecer o fluxo de sangue (Oliveira et al., 2018; Nascimento et al., 2021).

Para realização da técnica, pode se utilizar via radial ou femoral, contundo, os médicos especialistas costumam pela via radial, por proporcionar conforto ao paciente, porém, continua sendo primeira escolha, a femoral, por ser mais confiável, entretanto, exige um período de repouso a mais e longa permanência no hospital (Soares et al., 2017; Teixeira et al., 2019).

É importante que o paciente fique totalmente em repouso aos cuidados da equipe de enfermagem na sala de recuperação a fim de evitar instabilidade, na via femoral, o cliente deve ficar em decúbito dorsal com membro que foi realizado o cateterismo, reduzido riscos de coágulos sanguíneos (Sartori et al., 2018; Silva et al., 2018).

Alguns riscos podem acontecer, sendo competência da enfermagem em monitorar esses sinais como sangramento ou infecção do local, lesão nos vasos sanguíneos, reação alérgica por conta do contraste, arritmias, tromboembolismo e pressão arterial alterada (Alencar et al., 2021; Castro et al., 2016).

Em média, a técnica dura 30 minutos, longo, existe todo o processo de preparação ao repouso do paciente, como jejum de 4 horas antes do exame, descanso e uso de medicamentos orientados pelos cardiologistas, evitando chás, remédios ou medicamentos duvidosos (Aguiar et al., 2016).

A assistência de enfermagem deve pauta-se em processo contínuo e ininterrupto, podendo ser dividido no pré, perioperatório e pós-operatória, tendo essa divisão para que seja compreendido o todo diante das ações de enfermagem. No pré-operatório mediato, antes da internação, e imediata que antecede as horas da intervenção cirúrgica. A enfermagem deve fazer parte da preparação psicológica e na diminuição da ansiedade do paciente a fim diminuir eventos adversos, deve firmar uma interação entre equipe de enfermagem e paciente, orientando cuidados, supervisionado e identificado problemas futuros (Silva et al., 2016).

É importante que enfermagem se atente a segurança do paciente, tema esse discutido há tempos, que visa redução de riscos associados ao cuidado da saúdE, evitando ou prevenidas consequências ao curto ou longo prazo decorrente do processo 
em saúde, aplicado ao paciente com cateterismo, é necessário que esteja atento manipulação asséptica da ferida operatóriA e monitorar sinais logísticos (Castro et al., 2016).

Diante desse cenário, destaca-se a SAE, o processo de enfermagem que visa melhorar e conceituar todo trabalho de enfermagem, organizado e facilitado sua comunicação com cliente e equipe multidisciplinar, garantido o trabalho contínuo do cuidado e assegurado de forma jurídica, por meio de anotações e registro tudo que foi realizado com paciente (Silva et al., 2018).

A consulta de enfermagem começa no primeiro contato do paciente na hemodinâmica, onde o enfermeiro tem por obrigação coleta informações importantes, por exemplo, os sentimentos e angústias do paciente e dos familiares, histórico pregressa e hábitos diários a fim de desenvolver ações que possa trazer conforto, além de diminuir riscos associado ao contraste iodado e exposições (Nascimento et al., 2021).

A consulta de enfermagem no período pré e pós exames possibilita ao enfermeiro efetuar o diagnóstico adequado e preparar uma assistência mais qualificada aos clientes, impedindo intercorrências e interrupções no procedimento, favorecendo maior comodidade, atuando positivamente para a manutenção da qualidade de vida dos pacientes. Através do diagnóstico de enfermagem, o profissional deve se atentar para o uso adequado do NANDA, efetuando um julgamento devidamente clínico. Para que seja elaborado um diagnóstico exato, o enfermeiro deve atentar para a complicação de saúde encontrada e não fugir da investigação realizada. É importante também, que o enfermeiro se entente para o diagnóstico de risco, nos locais de punção. A prática constante do julgamento de enfermagem na área clínica é o que propícia a execução de um cuidado individualizado (Soares et al., 2017).

O planejamento ocorrerá quando o enfermeiro identificar o que possui de diagnóstico e planejar um conjunto de metas a serem alcançadas. Constatar e registrar as intervenções de curativos no espaço da punção, aplicar medicações que atenuem a dor, quando necessário e prestar apoio emocional quando notar a necessidade no período pós procedimentos (Nascimento et al., 2021.

Na elaboração de cuidados, é necessário que se observe as orientações ao longo do procedimento, reduzindo os sinais de ansiedade. Existem inúmeras maneiras de reduzir determinados sintomas, uma delas é explicar ao paciente acerca do exame. O conhecimento reduz a incerteza por parte dos pacientes acerca dos procedimentos, permitindo assim, maior esclarecimento. Quando a instrução se faz efetiva, os resultados são evidenciados de maneira positiva no final dos procedimentos. O enfermeiro, por fim, deve avaliar o auxílio prestado e o que precisará ser refeito (Castro et al., 2016).

Torna-se indispensável que a assistência prestada pelo enfermeiro seja de qualidade, implementando cuidados, tais como formular estratégias de conforto, tal como a utilização de coxins, auxílio na deambulação ou mudança de decúbito, proporcionando um ambiente acolhedor e um monitoramento intermitente dos sinais vitais (Alencar et al., 2021; Castro et al., 2016; Silva et al., 2016).

O processo do cuidar da enfermagem, encontra-se estruturado em quatro pilares: a enfermagem para, pelo, com e ao paciente. O cuidado permite que a assistência de enfermagem seja prestada para o indivíduo e não apenas para a sua enfermidade através de um olhar holístico, dessa forma é possível agilizar os diagnósticos, tal qual o tratamento de agravos de saúde potenciais e vigentes. Dessa forma, os cuidados de enfermagem podem minimizar os riscos de intercorrências através da observação continuada e intervenção antecipada, sendo crucial para a recuperação do paciente (Sartori et al., 2018).

A SAEP é de grande contribuição para a prevenção de agravos e complicações cirúrgicas, tal como é fundamental para o planejamento da assistência perioperatória. Por meio dela, a equipe de enfermagem é capaz de oferecer suporte tanto ao paciente quanto para sua família, no processo de compreensão da condição de saúde e de como o tratamento prosseguirá, possibilitando uma assistência integral, continuada, documentada e individualizada. 
Desta forma, os pacientes que outrora foram submetidos à cateterismo cardíaco, necessitam, por parte do enfermeiro, uma assistência sistematizada, do momento de entrada na unidade até sua alta. Sendo que, os cuidados de enfermagem devem se centrar no pré e pós operação, a fim de identificar e prevenir complicações. Os cuidados de enfermagem devem ser minuciosos, visto que, um simples quadro de dor local pode evoluir para uma elevação da pressão arterial, provocando sangramentos em locais de punção e, em alguns casos, incidir em uma complicação mais severa (Silva et al., 2018).

A equipe de enfermagem possui grande valor no processo de acompanhamento e monitorização, tendo em vista que a mesma é responsável pelo preparo prévio do material, bem como pela manutenção da monitorização e atua veementemente no processo de prevenção de agravos por meio da observação de sinais de risco. Além disso, cabe ao enfermeiro ser capaz de prover e prever os materiais e equipamentos necessários para a manutenção do estado geral de saúde do paciente conhecendo toda a técnica, indagar quando encontrar falhas e ser devidamente capaz de explicar os procedimentos para o paciente a fim de reduzir seu nível de stress e ansiedade, além de conseguir total colaboração (Soares et al., 2017).

Diante às mudanças na maneira de se prestar cuidados que acontecem de maneira continuada, o profissional da enfermagem é constantemente desafiado a conservar sua competência técnica, comprovando a forma como sua assistência pode afetar de maneira direta e positiva a evolução do paciente e ratificando sua participação ativa na tomada de decisões clínicas e na melhora da assistência. Neste âmbito, o enfermeiro é indispensável para a recuperação do paciente, trabalhando em equipe de maneira responsável. Seu trabalho deve ser fundamentado nos conhecimentos técnico-científicos, que são essenciais para coordenar um grupo que precisa estar treinado e apto para ajudar o paciente em todos as suas carências.

\section{Considerações Finais}

Sendo a equipe de enfermagem, o conjunto de profissionais mais presentes e com maior proximidade do paciente ao longo do processo de internação hospitalar, torna-se de sua responsabilidade a identificação e prevenção de agravos e complicações da condição de saúde de seus pacientes, bem como, a aplicação das intervenções necessárias para resolver os eventuais problemas. Em relação as habilidades e competências dos enfermeiros, é necessário um aprimoramento constante, em prol de outros métodos que vão sendo implantados a fim de se obter uma melhor relação profissional-paciente.

O paciente que necessita de uma intervenção cirúrgica, em muitas ocasiões se encontra em estado de ansiedade e receoso em virtude do ato, nessas situações, compete ao enfermeiro possuir a capacidade de prover os cuidados no pré e pósoperatório com o intuito de amenizar, ou mesmo eliminar, as dúvidas dos pacientes, bem como as de seus familiares essas estratégias são de grande valor para que se possa conduzir da maneira mais eficiente possível a recuperação dos pacientes.

Essa pesquisa envolveu estudo de vários livros e artigos sobre o tema, concluindo-se que é de fundamental importância para reabilitação do paciente aos cuidados dos enfermeiros. Assim, pode-se dizer que uma intervenção precoce do enfermeiro é capaz de minimizar e reduzir os agravos melhorando o conforto e a segurança do paciente.

Outros estudos posteriores podem fundamentar-se nas obrigações individuais dos enfermeiros que estão sendo intensamente enfatizados e julgados de maneira ética e as equipes estão cada vez mais ampliando os meios para proporcionar uma assistência com qualidade para os pacientes pautada na prevenção de possíveis complicações que o paciente submetido ao cateterismo que requerem cuidados pós-operatórios.

\section{Referências}

Aguiar, B. F., Rinaldi, E. C. A., Cintho, L. M. M., Martins, C. L. da S., \& Zimmerman, M. H. (2016). <b>Importância dos cuidados de enfermagem no cateterismo cardíaco. Ciência, Cuidado E Saúde, 15(3), 460 - 465.

Alencar, J. L., da Silva, L. V. M., Macias, B. S. G., de Souza Cravo, D. D., dos Santos, L. P., da Silva Ferreira, J., \& Sardinha, D. M. (2021). Uma revisão integrativa dos diagnósticos de enfermagem mais evidenciados no cateterismo cardíaco. Revista Eletrônica Acervo Enfermagem, 14, e8948-e8948. 
Bertolini, S. R. F., dos Santos, S. V. M., da Silva, L. A., \& Robazzi, M. L. D. C. C. (2019). AVALIAÇÃO DO CONHECIMENTO DOS PACIENTES SUBMETIDOS AO CATETERISMO CARDÍACO E ANGIOPLASTIA CORONÁRIA: UMA CONTRIBUIÇÃO PARA A ATUAÇÃO DA ENFERMAGEM. Revista Expressão Católica Saúde, 4(2), 44-51.

de Andrade Marconi, M., \& Lakatos, E. M. (2017). Metodologia do trabalho científico.

de Oliveira Castro, Y. T. B., Rolim, I. L. T. P., Silva, A. C. O., \& Silva, L. D. C. (2016). Conhecimento e significado do cateterismo cardíaco para pacientes cardiopatas. Rev Rene, 17(1), 29-35.

de Pádua, E. M. M. (2019). Metodologia da pesquisa: abordagem teórico-prática. Papirus Editora.

da Silva, V. F., Lohmann, P. M., Pissaia, L. F., \& da Costa, A. E. K. (2018). Cateterismo cardíaco: A entrevista frente aos anseios do paciente. Research, Society and Development, 7(12), e12712500-e12712500.

dos Santos Silva, M. A., Marques, P. T. V., de Castro, D. F. A., Padula, M. P. C., Yano, K. T., de Lima Coimbra, A. L., \& do Carmo, V. L. (2016). Relação entre orientação, ansiedade e dor em pacientes submetidos ao cateterismo cardíaco/Relation between orientation, anxiety and pain in cardiac catheterization. Arquivos Médicos dos Hospitais e da Faculdade de Ciências Médicas da Santa Casa de São Paulo, 61(1), 28-34.

Kuhn, O. T., Bueno, J. F. B., Loro, M. M., Kolankiewicz, A. C. B., Winkelmann, E. R., \& Rosanelli, C. L. P. (2015). Perfil de pacientes submetidos a cateterismo cardíaco e angioplastia em um hospital geral. Revista Contexto \& Saúde, 15(29), 4-14.

Machado, J. P., Franco, C. P., Pertuzzatti, J. L., Marartinelli, J. C., \& Welfer, M. (2017). Complicações do cateterismo cardíaco. SEFIC 2017.

Maciel, B. S., Barros, A. L. B. L. D., \& Lopes, J. D. L. (2016). Elaboração e validação de um manual informativo sobre cateterismo cardíaco. Acta Paulista de Enfermagem, 29, 633-642.

Moreira, M. L. A. P., Mizuno, E., \& Meireles, G. C. X. (2017). Consulta de enfermagem pré-cateterismo cardíaco e intervenções coronárias percutâneas. Rev. enferm. UFPE on line, 2548-2556.

Nascimento, R. K. M., Andrade, K. B. S., Camerini, F. G., Franco, A. S., Marins, A. L. C., \& Naves, C. B. D. O. C. (2021). Consulta de enfermagem préprocedimento de cateterismo cardíaco: avaliação da satisfação do paciente. Revista Enfermagem UERJ, $29,49970$.

Oliveira, A. A., Viana, C. P., da Silva, É. P. B., Maia, J. S., Pereira, M. J. B., Venturi, V., \& dos Santos Maia, L. F. (2018). O cateterismo cardíaco e a enfermagem: a importância dos diagnósticos de enfermagem para uma assistência de qualidade. Revista Recien-Revista Científica de Enfermagem, 8(23), 2127.

Pereira, A. S., Shitsuka, D. M., Parreira, F. J., \& Shitsuka, R. (2018). Metodologia da pesquisa científica. de Andrade Marconi, M., \& Lakatos, E. M. (2017). Metodologia do trabalho científico.

Régis, A. P., Dalla Rosa, G. C., \& Lunelli, T. (2017). Cuidados de enfermagem no cateterisno cardíaco e angioplastia coronariana: desenvolvimento de um instrumento. Revista Recien-Revista Científica de Enfermagem, 7(21), 3-20.

Sartori, A. A., Gaedke, M. Â., Moreira, A. C., \& Graeff, M. D. S. (2018). Diagnósticos de enfermagem no setor de hemodinâmica: uma perspectiva adaptativa. Revista da Escola de Enfermagem da USP, 52.

Severino, A. J. (2017). Metodologia do trabalho científico. Cortez editora.

Soares, M. M. D. S., Alencar, F. I. L. D., Osterne, L. F. D. A., Florêncio, R. S., Pessoa, V. L. M. D. P., \& Cestari, V. R. F. (2017). Cateterismo cardíaco via femoral: descrição clínica e complicações associadas. Rev. enferm. UFPE on line, 1473-1480.

Teixeira, T. R. F., Avila, M. A. G. D., \& Braga, E. M. (2019). Compreensão de pacientes às orientações de enfermagem no cateterismo cardíaco: uma pesquisa qualitativa. Cogitare enferm, 24, e56604. 